\title{
The Aquarius ocean salinity mission high stability L-band radiometer
}

\author{
Fernando A Pellerano, Jeffrey Piepmeier, \\ Michael Triesky, Kevin Horgan, Joshua Forgione, \\ James Caldwell \\ Goddard Space Flight Center \\ Greenbelt, Maryland USA \\ Fernando.A.Pellerano@nasa.gov
}

\author{
William J Wilson, Simon Yueh, Michael Spencer, \\ Dalia McWatters, Adam Freedman \\ Jet Propulsion Laboratory \\ California Institute of Technology \\ Pasadena, California USA
}

\begin{abstract}
The NASA Earth Science System Pathfinder (ESSP) mission Aquarius, will measure global ocean surface salinity with $\sim 120 \mathrm{~km}$ spatial resolution every 7-days with an average monthly salinity accuracy of $0.2 \mathrm{psu}$ (parts per thousand) [1]. This requires an $L$-band low-noise radiometer with the long-term calibration stability of $\leq 0.15 \mathrm{~K}$ over 7 days. The instrument utilizes a push-broom configuration which makes it impractical to use a traditional warm load and cold plate in front of the feedhorns. Therefore, to achieve the necessary performance Aquarius utilizes a Dicke radiometer with noise injection to perform a warm - hot calibration. The radiometer sequence between antenna, Dicke load, and noise diode has been optimized to maximize antenna observations and therefore minimize NEDT. This is possible due the ability to thermally control the radiometer electronics and front-end components to $0.1{ }^{\circ} \mathrm{Crms}$ over 7 days.
\end{abstract}

Keywords-radiometer; L-band radiometry; salinity; stable radiometers

\section{INTRODUCTION}

The radiometers are Dicke radiometers that use noise injection for calibration. A critical requirement in the design of these radiometers is long-term (several days) stability. Stability is critical because significant averaging must be done to achieve the Aquarius goal for an accuracy of $0.2 \mathrm{psu}$ (gobal $\mathrm{rms}$ on a monthly basis). Since it will take 7 days to map the globe, the radiometers must be stable over at least 7 days. The design requirement set for Aquarius was that the radiometers be stable to within $0.13 \mathrm{~K}$ over 7 days. A primary element in maintaining stability is adequate internal calibration and good thermal control. The design adopted for the Aquarius radiometers is based on research conducted under NASA's Instrument Incubator Program (Ultra-Stable Radiometers, [2]) that uses two internal reference sources (noise diode and Dicke load).

\section{RADIOMETER CONFIGURATION}

The radiometer is divided into several elements as shown in Fig. 1 and Fig. 2. Beginning at the OMT, these elements are the OMT Couplers, the Correlated Noise Diode (CND), two diplexers (one for each polarization), the Radiometer Front End (RFE), the Radiometer Back End (RBE), and the Digital
Processing Unit (DPU). The OMT Couplers, CND, diplexers, and RFE are mounted on the OMT as shown in Fig. 1. Thermal control of these elements is critical to obtaining the radiometric stability needed for successful retrieval of salinity at the accuracy desired for Aquarius. The OMT assembly is cold-biased passively by means of radiator plates and appropriate coatings and actively controlled using heaters mounted to the assembly. The design requirement is to control the thermal environment to change less than $0.1^{\circ} \mathrm{C}$ over 7 days.

\section{RADIOMETER FRONT-END}

The primary amplification is done in the radiometer front ends (RFE). There is a separate RFE for each feed assembly. In the RFE, the two signals from the OMT (one for vertical polarization and one for horizontal polarization) are amplified and then combined to form four channels (vertical, horizontal and the sum and difference which is equivalent polarization at \pm 45 degrees). The sum and difference signal will be used to compute the third Stoke's parameter (e.g. detected with a square-law detector in the RBE and later subtracted during the ground processing). The first elements at the input of the RFE are the Dicke switch and its reference load followed by a coupler to a noise diode that provides the hot load (Fig. 2). Together, these are the references used for internal calibration. These calibration references are at the heart of the radiometer performance and tight thermal control is needed to meet the required radiometric performance. In order to meet the required performance, the radiometric temperature of the Dicke load must be known with an uncertainty of $<50 \mathrm{mK}$, and the coupled noise temperature must be stable to $<300 \mathrm{ppm}$. These parameters, coupled with the thermal control mentioned above, have been shown to be adequate to achieve the required radiometric stability (0.13K over 7 days; [2]). Also, this radiometer architecture is largely implemented via microstipbased technologies in order to reduce size and improve thermal control.

Between the OMT and the RFE are the OMT Coupler and the diplexers (Fig. 2). The OMT coupler provides a port for injecting signal from a second noise diode, the CND. This signal is used to monitor the phase and amplitude balance 


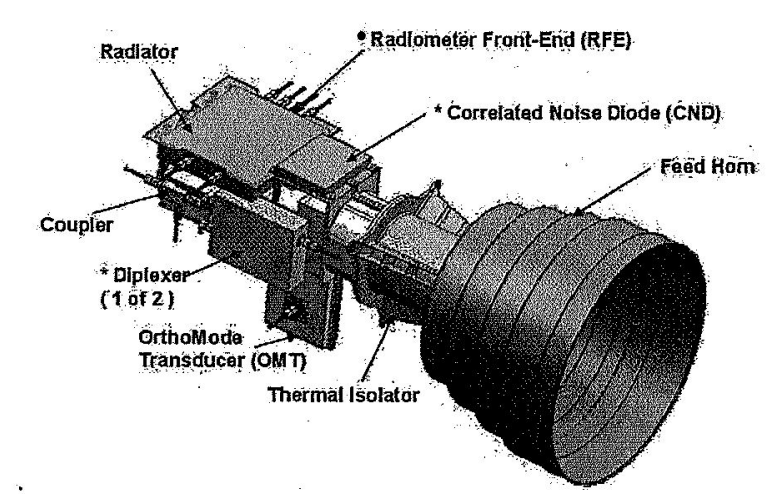

Figure 1. The radiometer front-end components are mounted around the Ortho-Mode Transducer to achieve the necessary thermal stability.

between the channels, a calibration that is necessary for proper polarimetric performance and calculation of the third Stoke's parameter. The diplexers are devices that allow the scatterometer and radiometer to use the same feed horn assembly without damage to the radiometer electronics. They are cavity type filters that provide enough rejection to guarantee no damage to the radiometer from the Aquarius scatterometer pulse. To provide additional isolation, whenever the scatterometer transmits the Dicke switches are switched to the reference load. The total isolation (diplexer plus switch) prevents any active component in the RFE from being saturated by the scatterometer.

\section{RADIOMETER BACK-END}

The radiometer back end (RBE) contains additional amplification, band-pass filtering, and the detectors for each channel (Fig. 2). Its performance (stability) is less critical to the overall stability of the radiometer system because it is located behind all the calibration sources and after the first stage gain in the RFE. The design requirement for temperature control of the RBE is a maximum change of $0.4 \mathrm{C}$ (rms) over 7 days. Because less control is necessary, the RBE has been physically separated from the more critical elements mounted on the OMT to facilitate the thermal control of this group.

The final stage of the radiometer is the digital processing unit (DPU). The detected signals from each radiometer channel are digitized in the RBE using voltage-to-frequency converters (VFC). These devices output pulses whose frequency is proportional to the detected signal. These pulses are counted asynchronously, and the frequency determined, by the DPU. The DPU also houses the radiometer controller and collects temperature and housekeeping data.

\section{RADIOMETER OPERATION}

Fig. 3 shows the timing diagram for the hardware. The fundamental timing unit for the hardware is $10 \mathrm{~ms}$ (approximately $1 \mathrm{~ms}$ for the scatterometer transmit pulse and 9 ms of observation time for the radiometer). The radiometer and scatterometer operation are alternated at a rapid rate so that the two sensors look at the same piece of ocean nearly simultaneously. The three radiometers (one for each beam) operate in parallel. During $120 \mathrm{~ms}$ each radiometer collects 7 samples (i.e. $9 \mathrm{~ms}$ long and repeated each $10 \mathrm{~ms}$ ) looking into the antenna followed by 5 samples devoted to the calibration sources (two noise diodes and Dicke load). This $120 \mathrm{~ms}$ sequence is then repeated. However, because of limitations with the on-board data storage, not all of this data will be downloaded. It is planned to average two of the antenna looks and transmit to ground three samples at the $10 \mathrm{~ms}$ resolution followed by two at $20 \mathrm{~ms}$. The samples of the calibration references transmitted to the ground will be the average of 10 samples.

The switching sequence between antenna, Dicke load, and noise diodes has been optimized to maximize antenna observations and minimize NEDT. This optimization is based on the fact that the radiometer gain and receiver temperature are relatively stable quantities. Therefore, the duty cycle of the calibration observations can be reduced to be consistent with the time constant of these parameters. The stable values needed for calibration are obtained by averaging over longer periods. The effect of this approach is a significantly higher duty cycle for the antenna (i.e., ocean) observations with an improved NEDT compared to the standard approach using equal duty cycles [2].

\section{RADIO FREQUENCY INTERFERENCE}

The radiometer has also been designed to include precautions against Radio Frequency Interference (RFI). A study conducted by NASA and ITT looked at potentially damaging sources, as well as interference that would impact the science retrieval [3]. The primary source of interference for the radiometer is ground-based air surveillance radars. The level of interference is a function of out-of-band emissions from these radars and also a function of the rejection level of radiometer filters in the skirts where the radar band is located (i.e. where the radar is permitted to transmit). The findings of the study suggested a three-tiered approach. The first step is to include 2 watt limiters to protect the low noise amplifiers (LNA) in the RFE against worst case damage. The second approach is to sample at sufficiently high rate to facilitate identification of RFI with the ability to remove it without complete loss of data. The study suggested that the radiometer data should not be averaged more than about $30 \mathrm{~ms}$ before download. The current design calls for most data to be down loaded at $10 \mathrm{~ms}$ sample rate and some at $20 \mathrm{~ms}$. Finally, the radiometer band-limiting filters (located in the RBE) were designed to reduce the potential interference to a probability of $<0.1 \%$ over the ocean. In terms of filter parameters, this requirement is equivalent to a $25 \mathrm{MHz}$ bandwidth, 7-pole Chebyshev filter. This is achieved with several filter stages. These include the diplexer and the two band pass filters in the RBE before the detector (Fig. 2). 


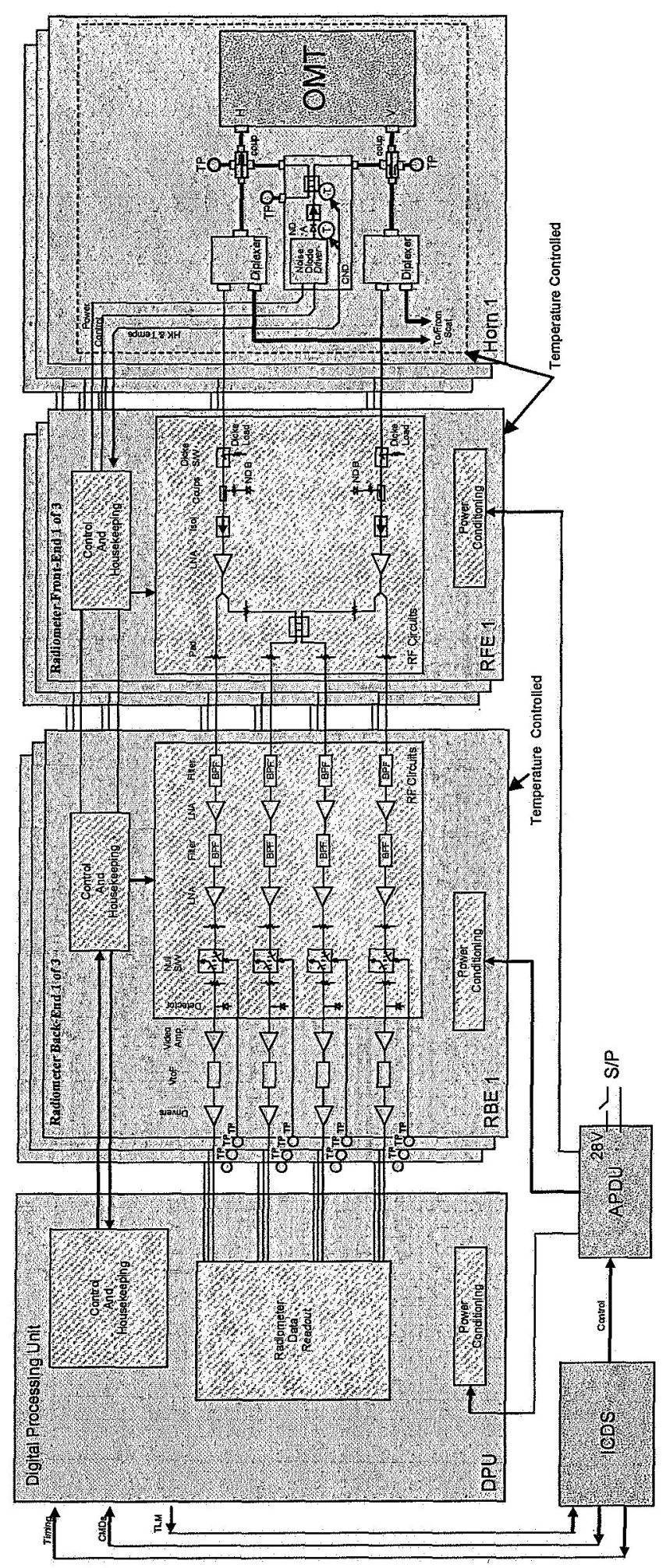

Figure 2. The radiometer is subdivided into the front-end, back-end, and digital processing unit as shown in this block diagram. 


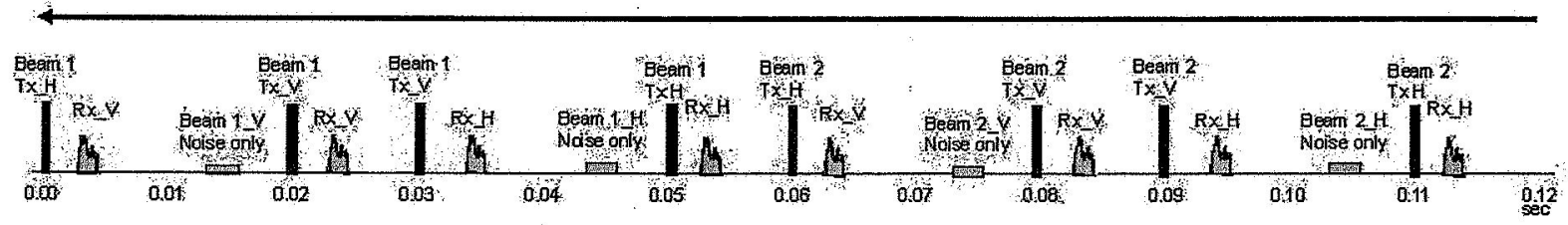

One Aquaris scattemeter Timing cycle, 180 ms

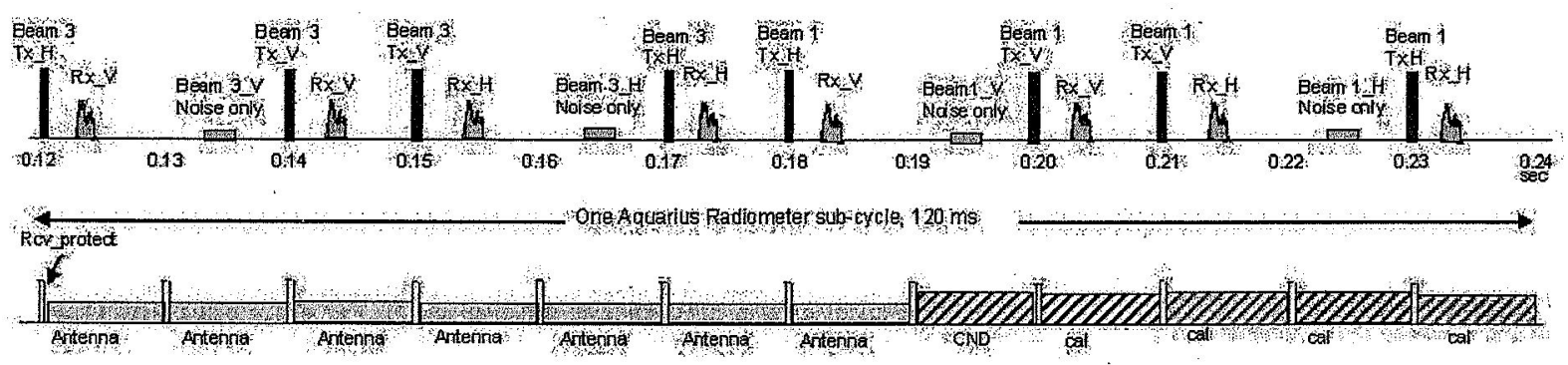

Figure 3. The system timing allows for simultaneous operation of the Aquarius radiometer and scatterometer.

\section{ACKNOWLEDGMENT}

The work described herein has been possible due to the contributions of many people in the Aquarius project. The authors would like to acknowledge all their team members at NASA's Goddard Space Flight Center and Jet Propulsion Laboratory.

\section{REFERENCES}

[1] D.M. Le Vine, G.S.E. Lagerloef, R. Colomb, S. Yueh, F. Pellerano "Aquarius: An Instrument to Monitor Sea Surface Salinity from Space", Submitted IEEE Trans. Geosci. \& Remote Sens.

[2] W. J. Wilson, A. Tanner, F. Pellerano, and K. Horgan, Ultrastable Radiometers for Future Sea Surface Salinity Missions, Jet Propulsion Laboratory Report D-31794, April 2005. 15.

[3] J. R. Piepmeier, F. A. Pellerano, A. Freedman, "Mitigation of Terrestrial Radar Interference in L-Band Spaceborne Microwave Radiometers", International Geoscience and Remote Sensing Symposium, IGARSS06, Denver, CO, July, 2006. 


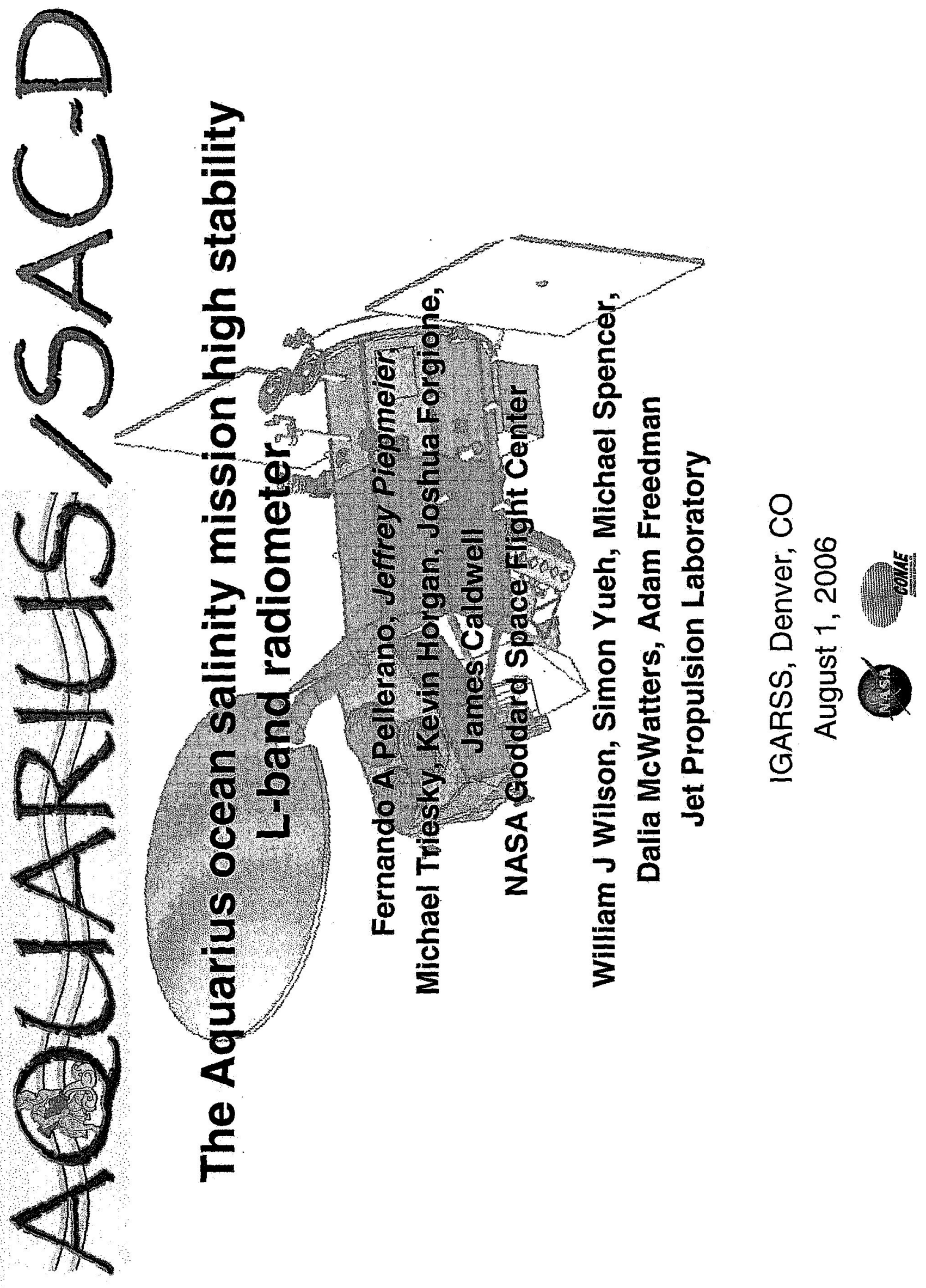




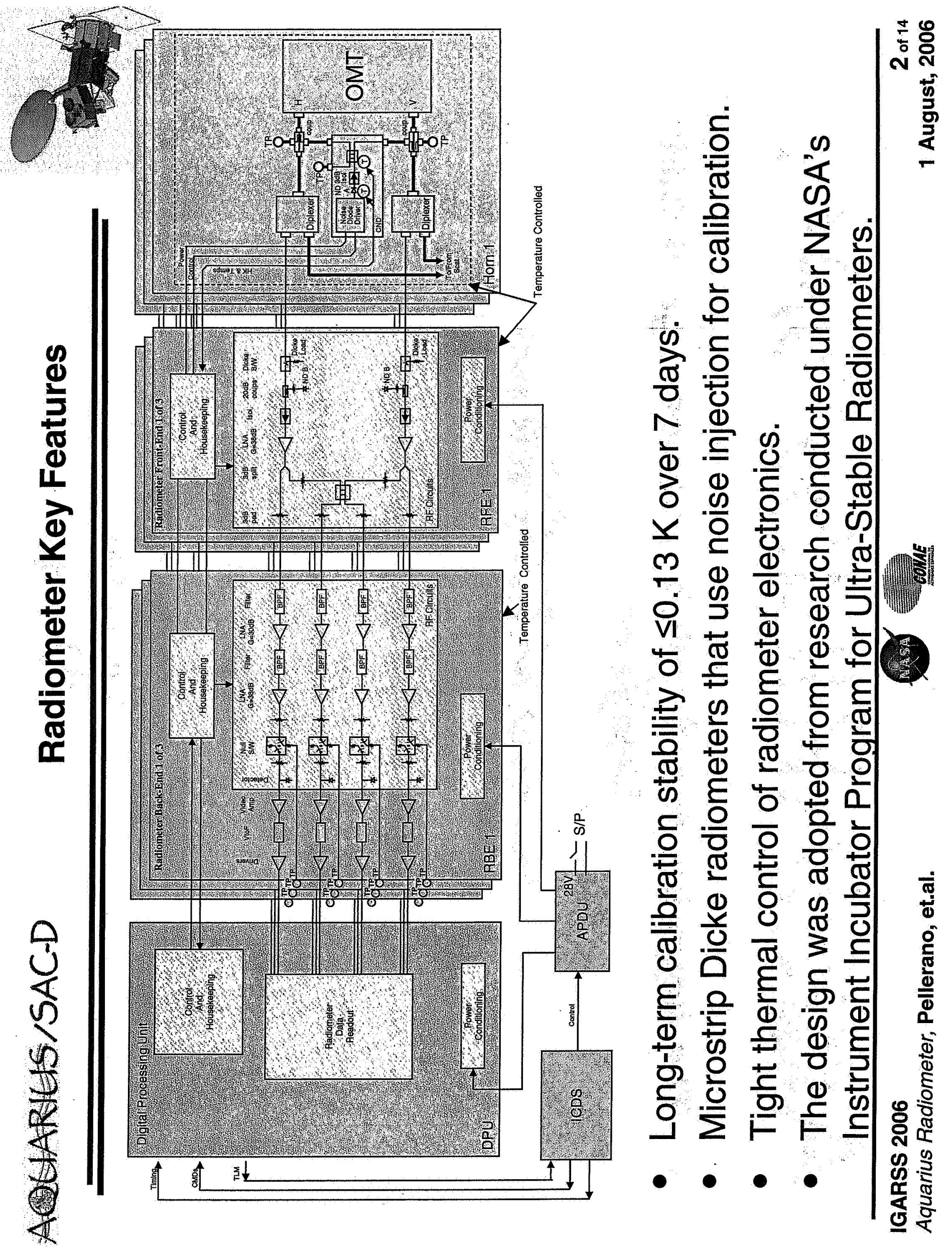




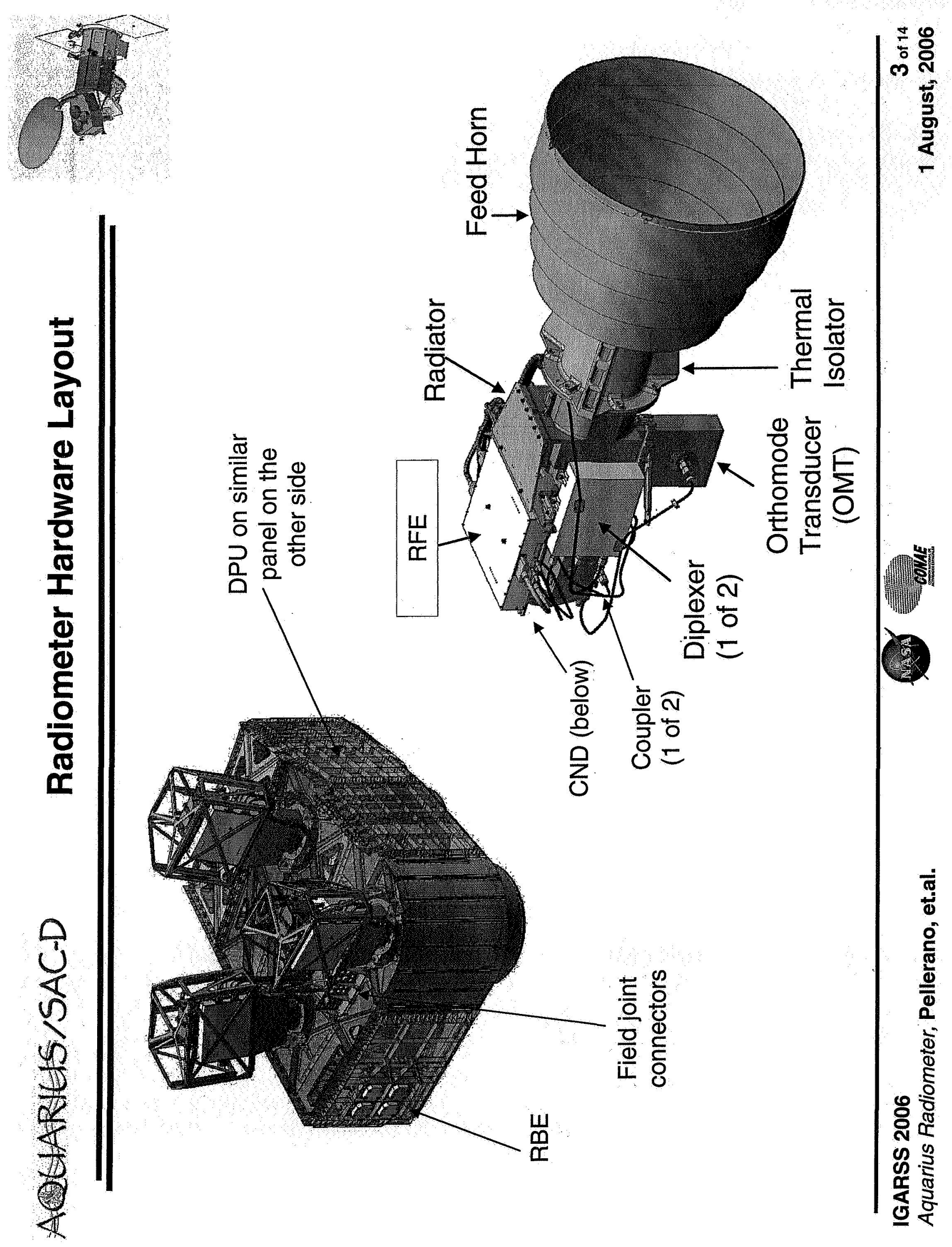




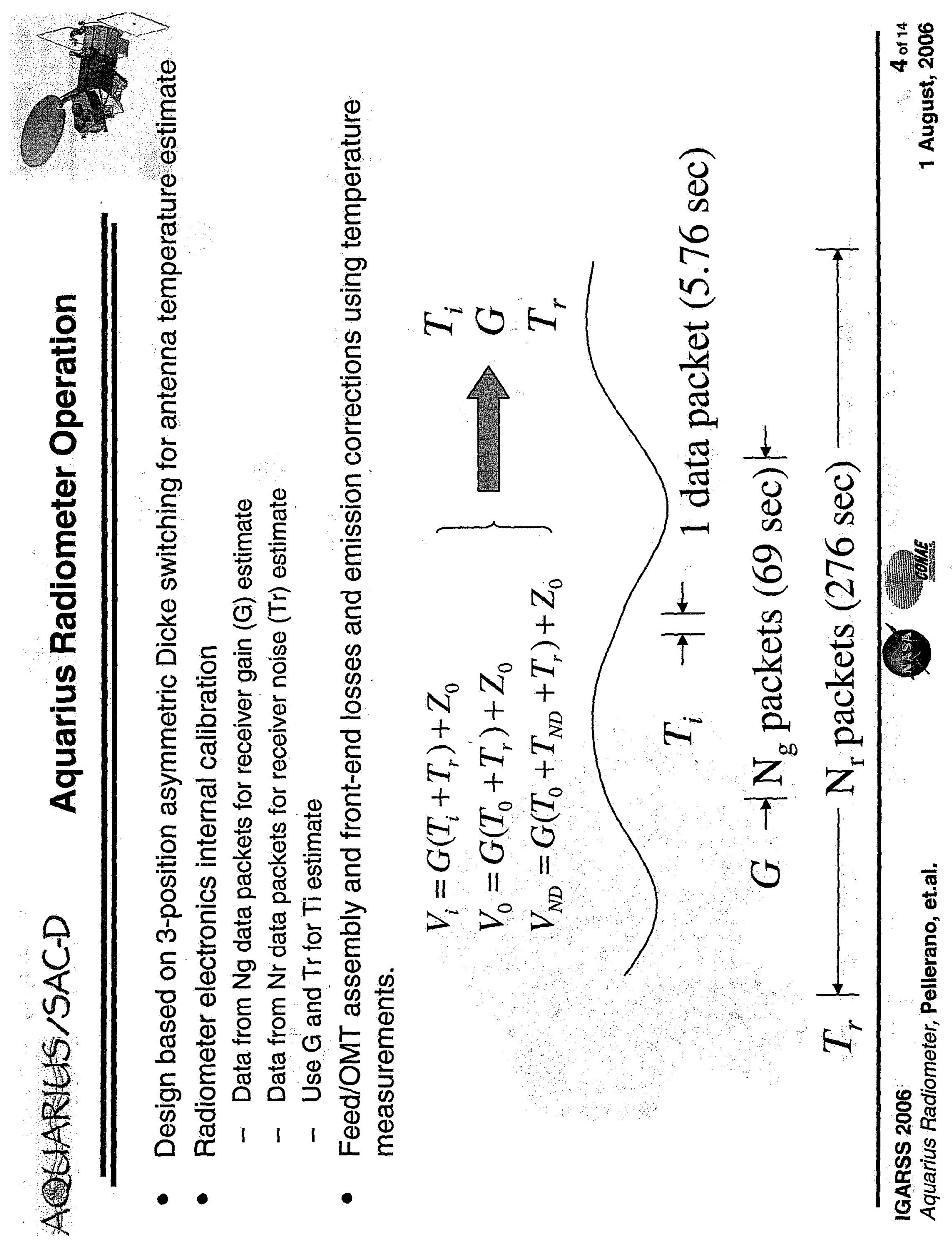



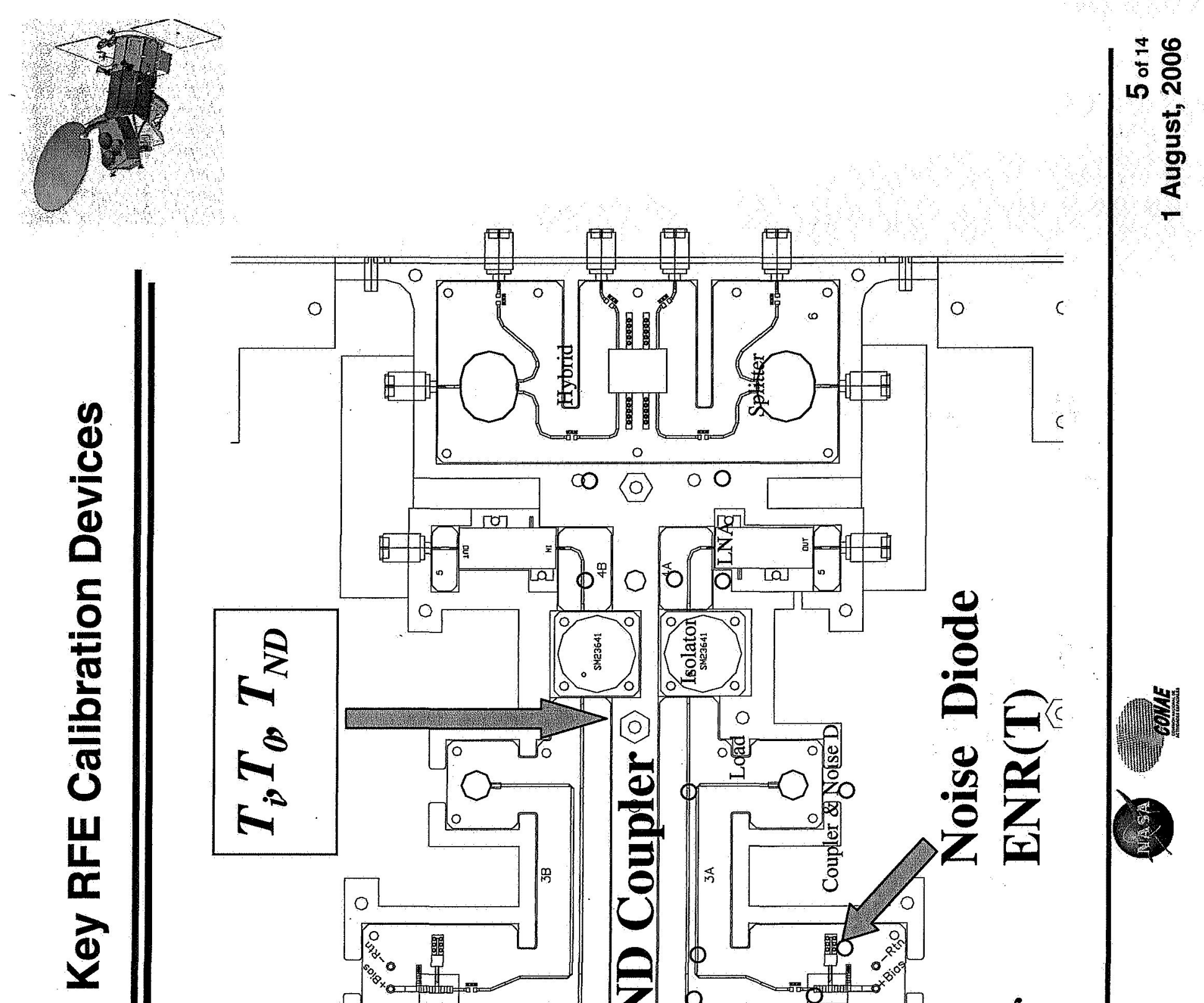



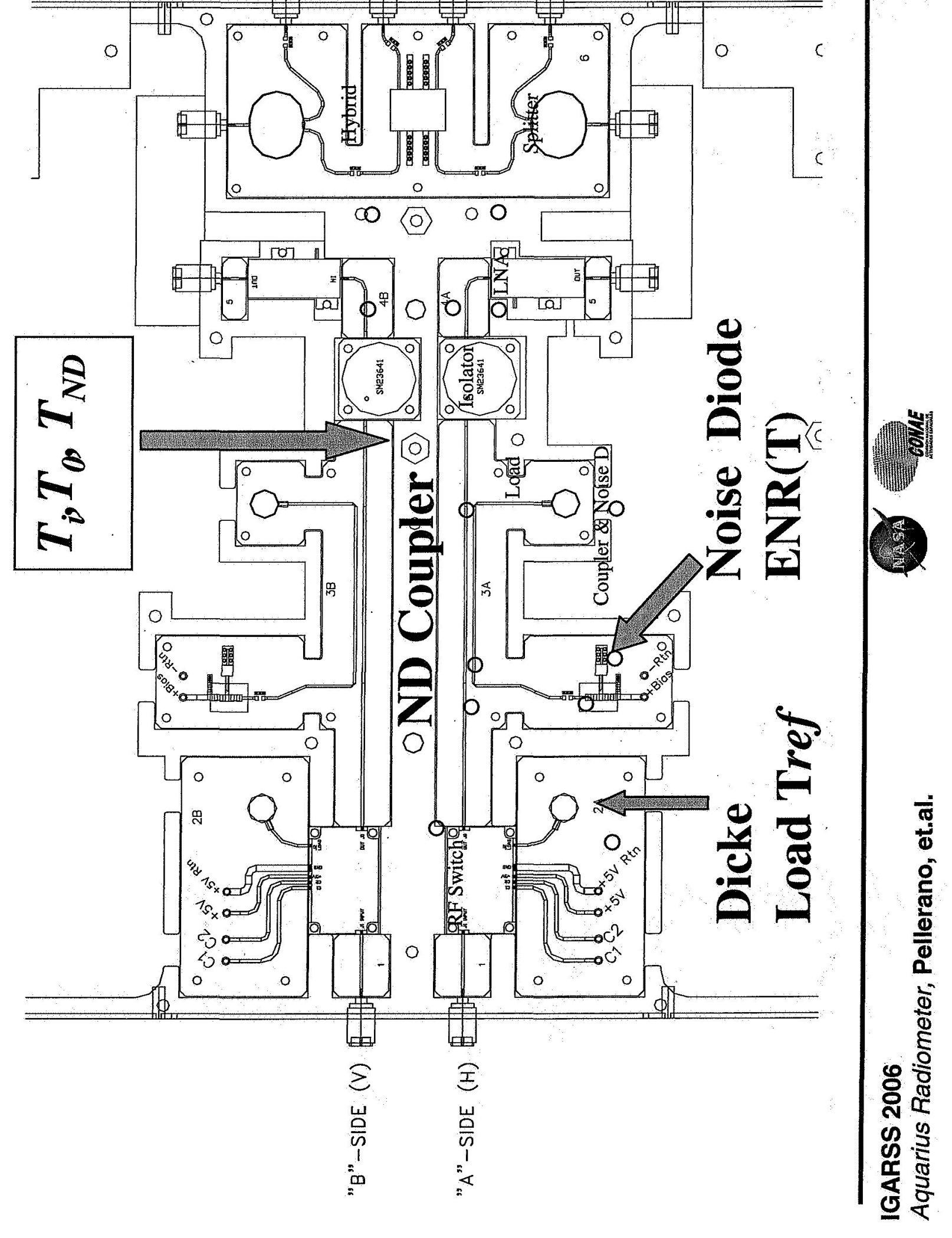




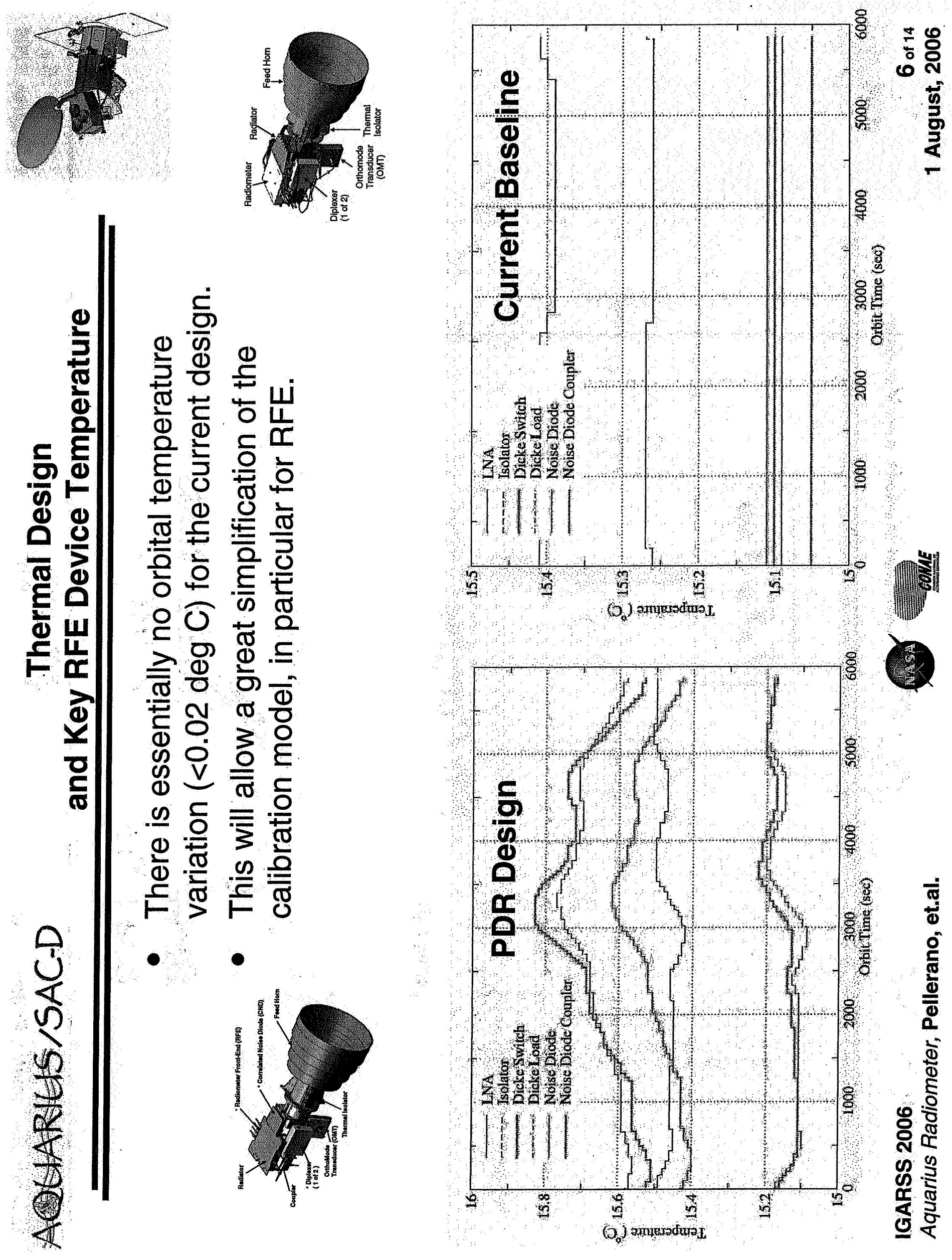




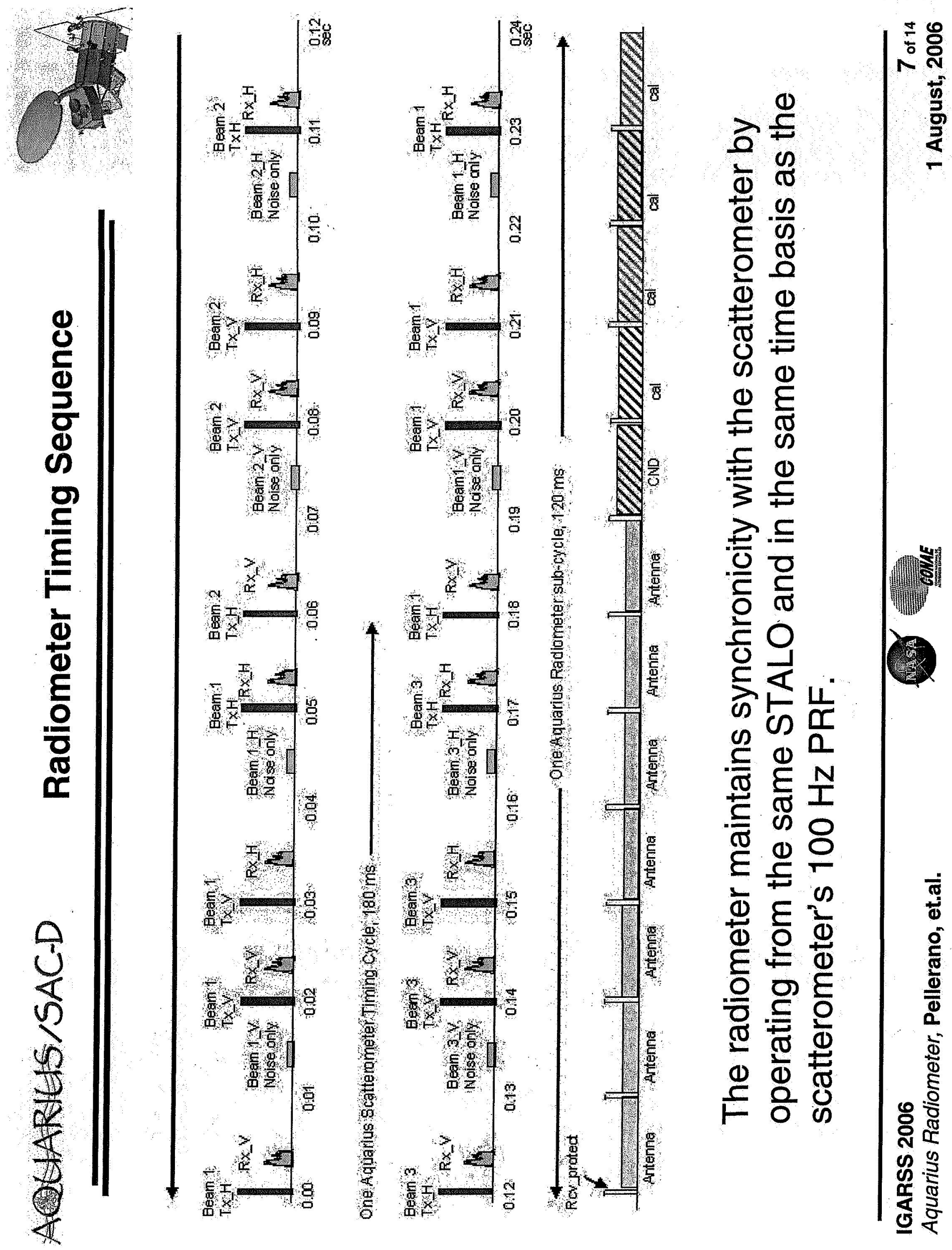




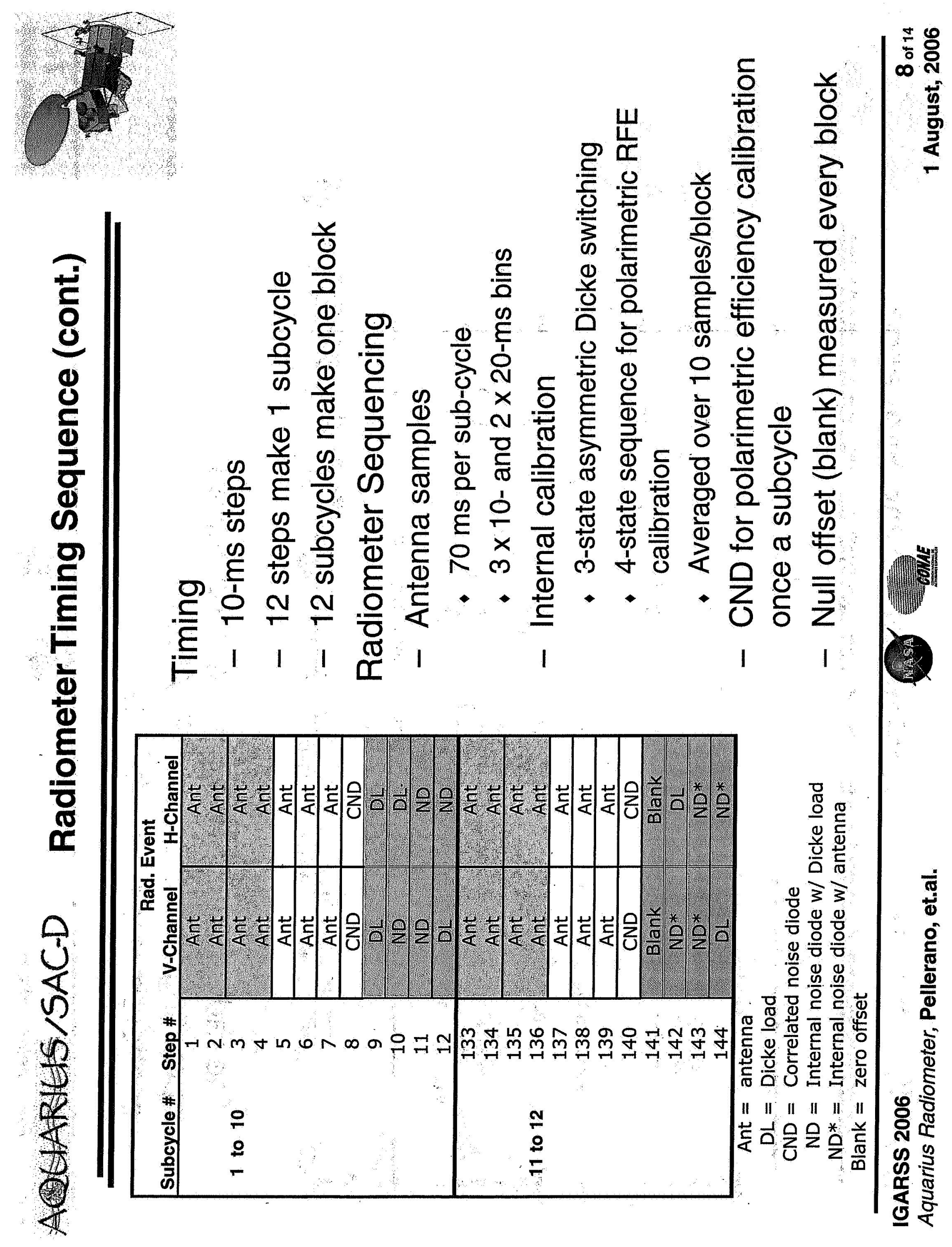




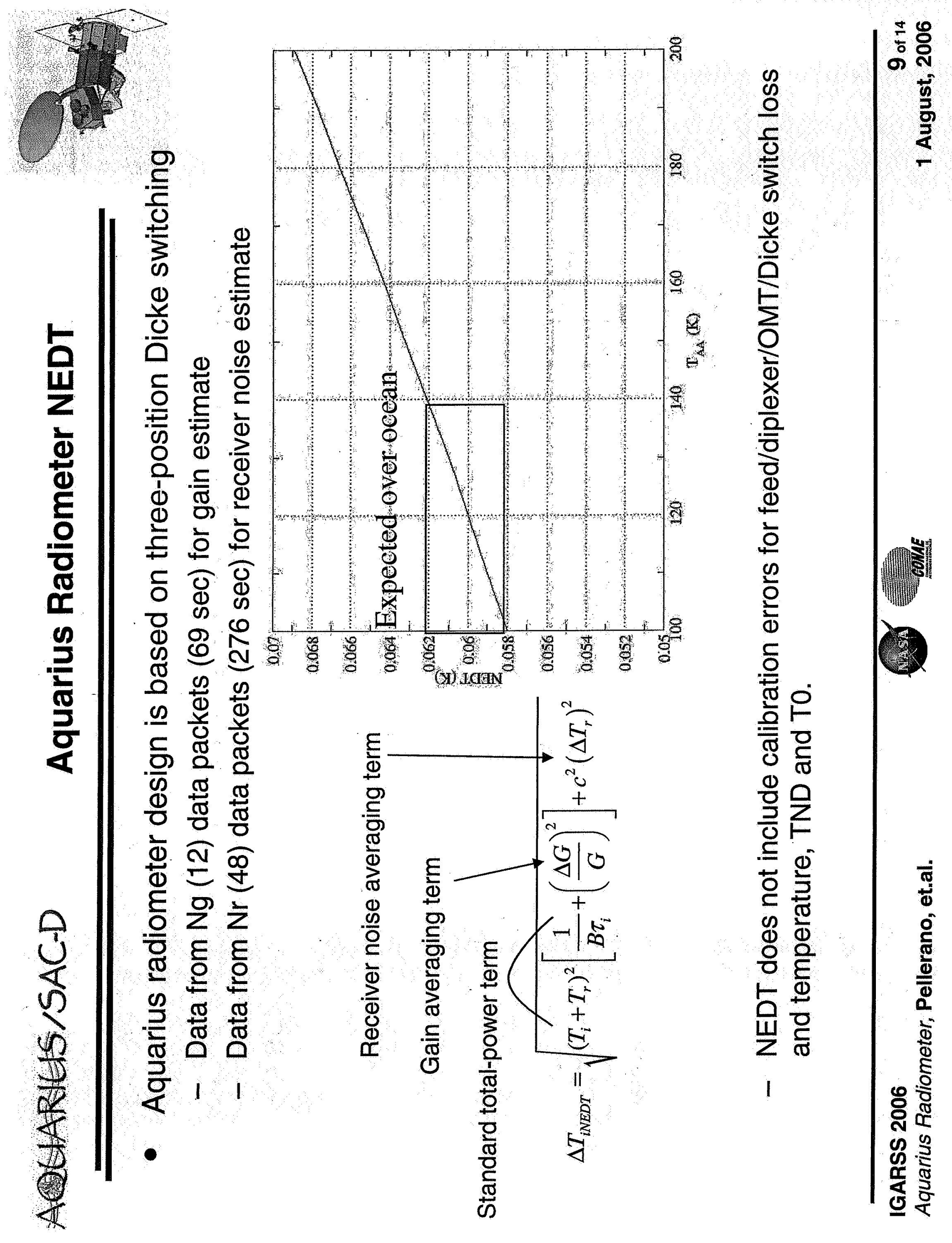




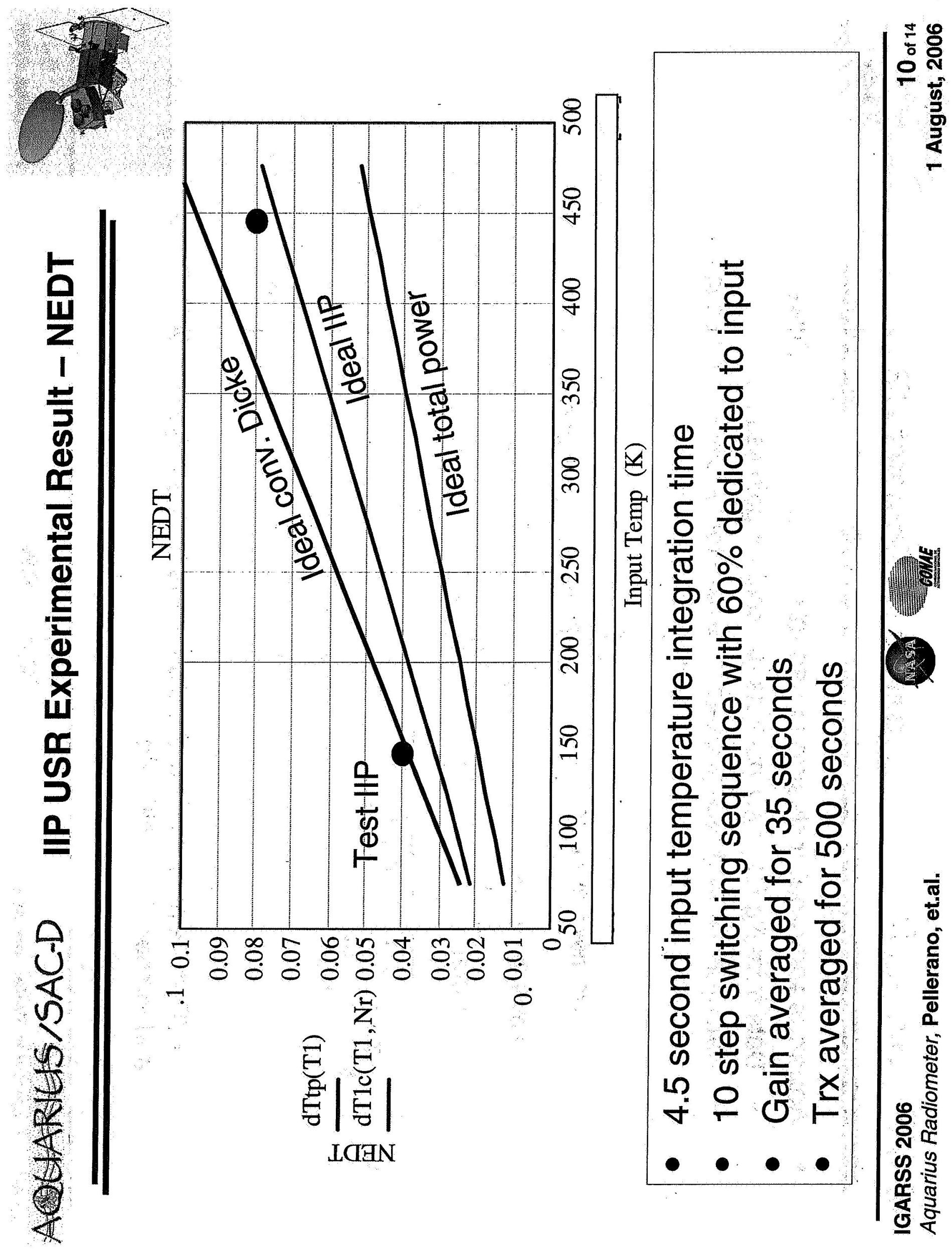



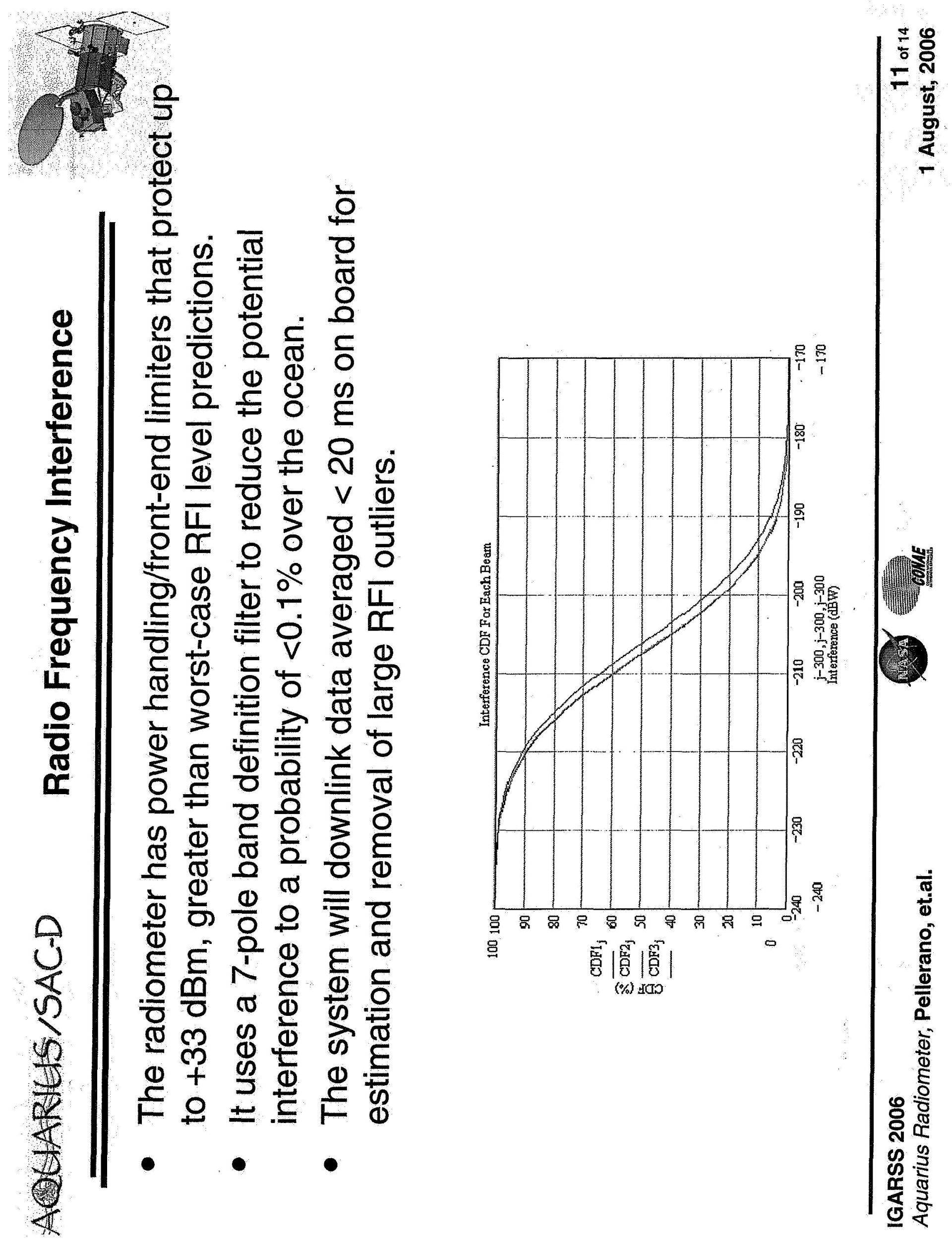


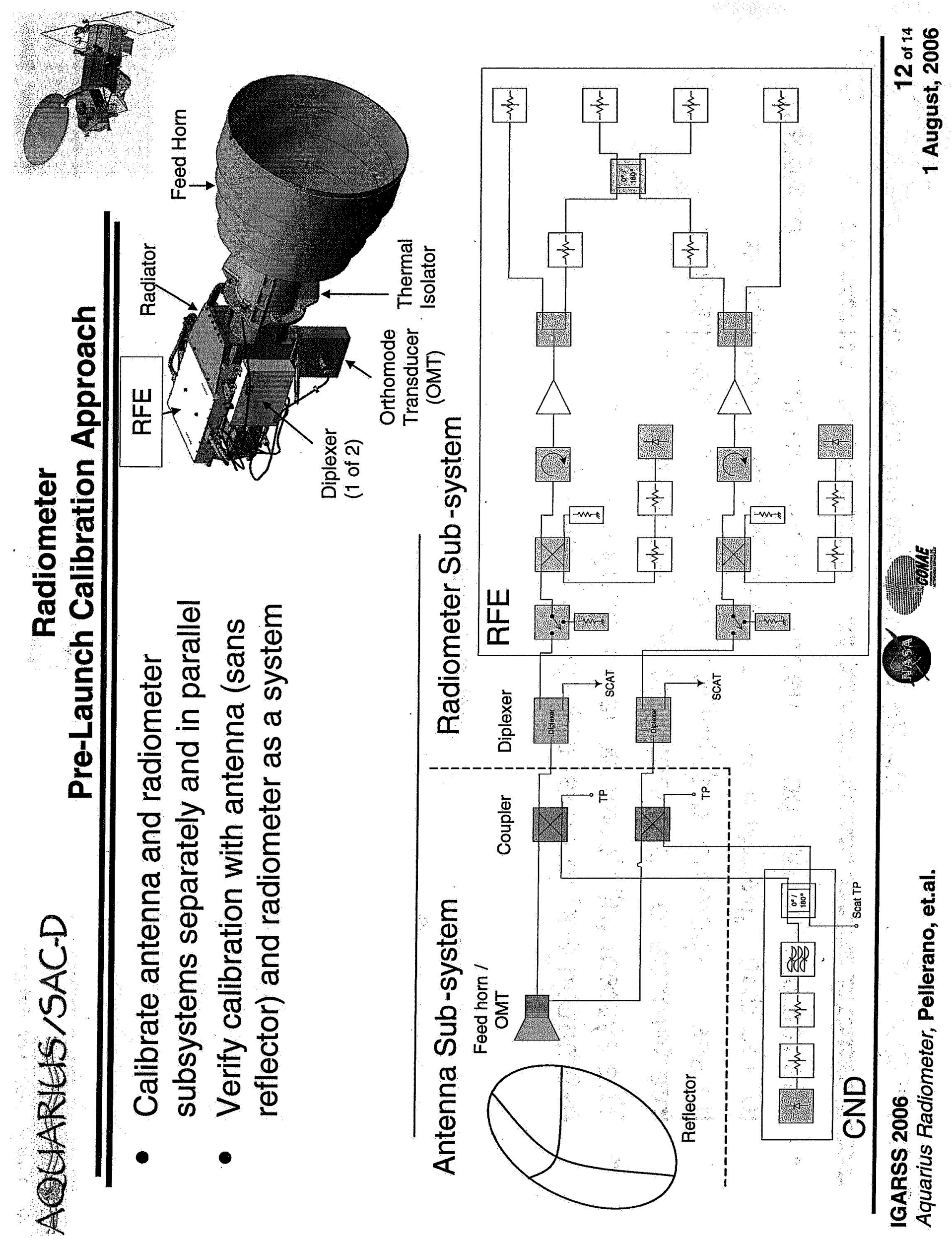




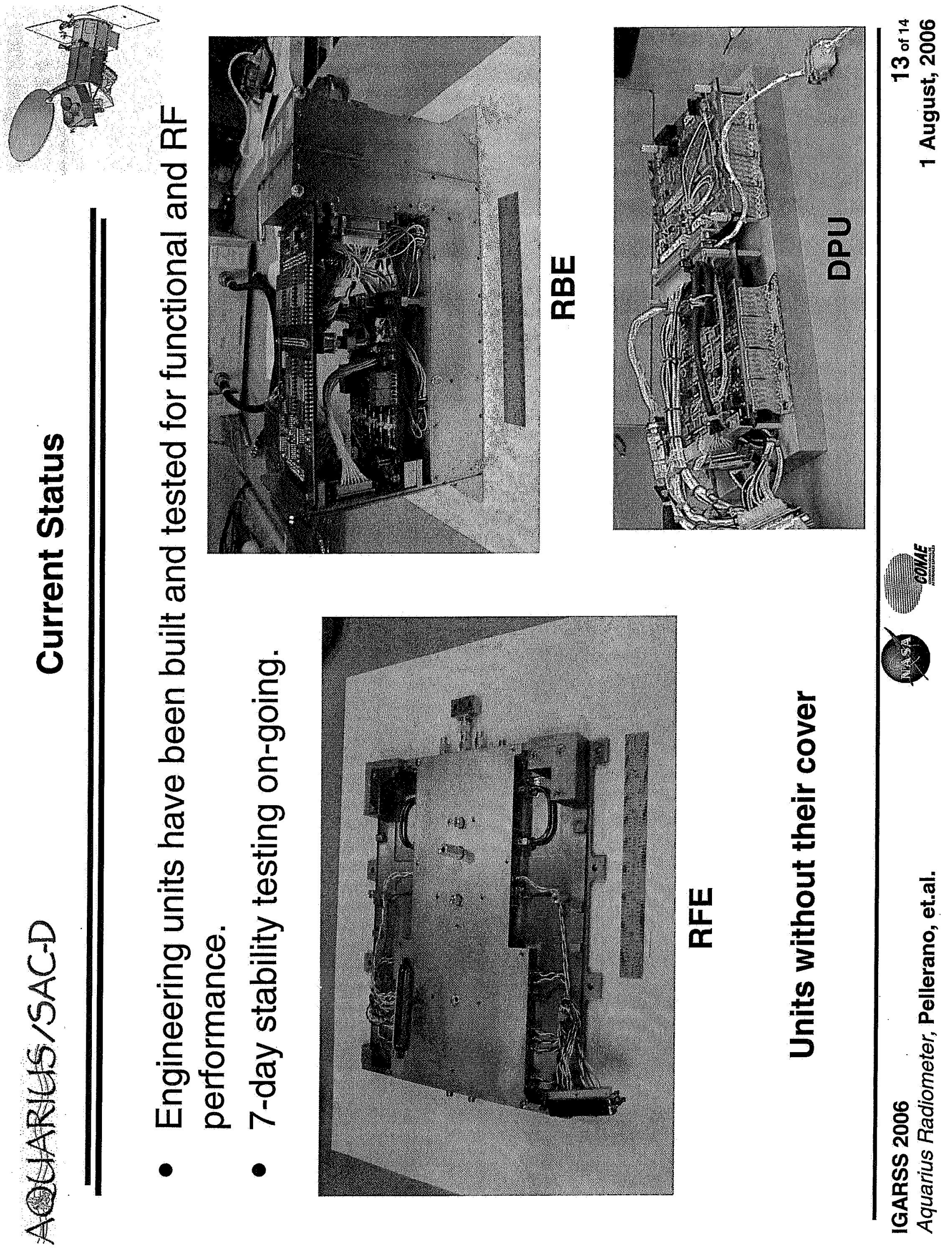




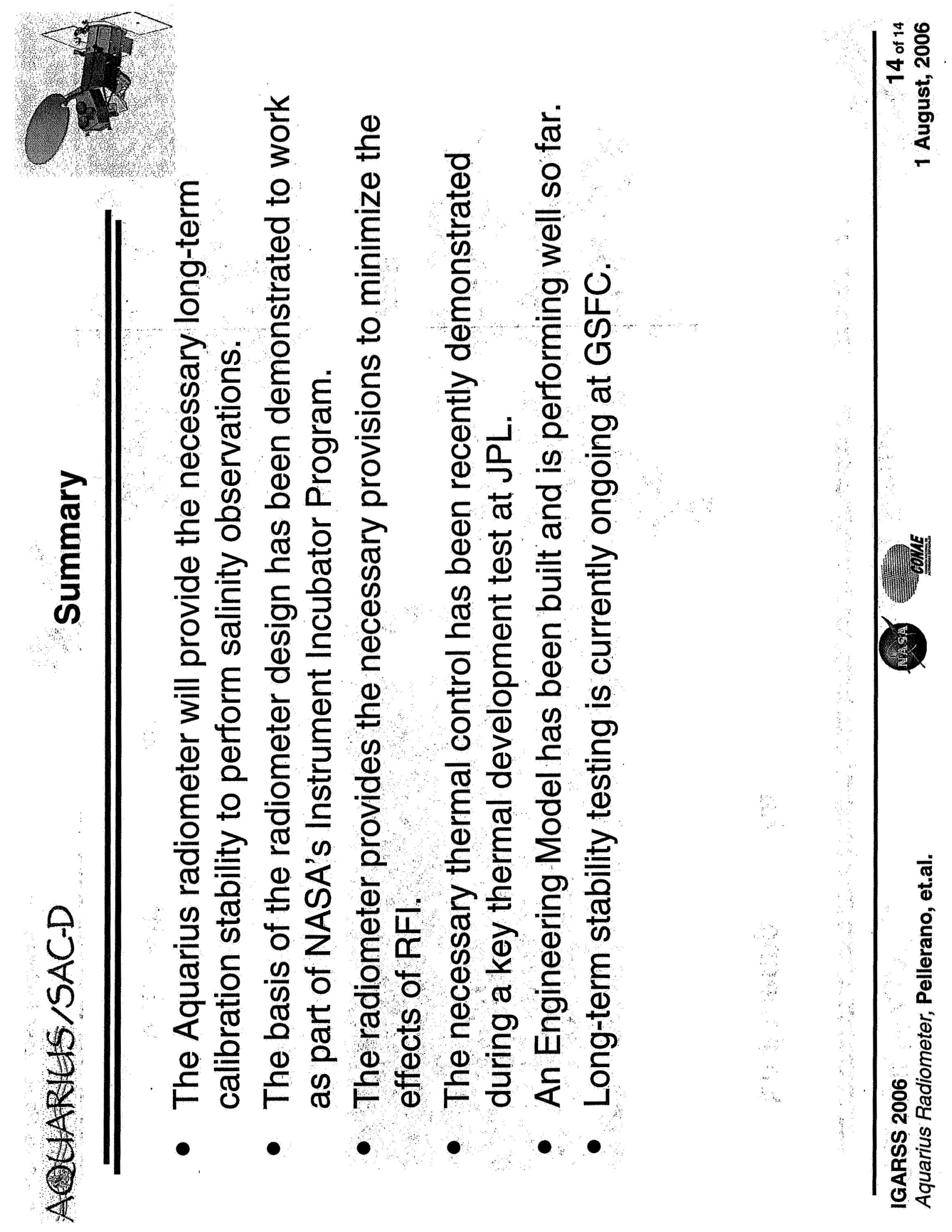

